\title{
EL PODCAST COMO MATERIAL DIDÁCTICO: PROCESO DE PRODUCCIÓN
}

Claudia Fabiola Ortega Barba

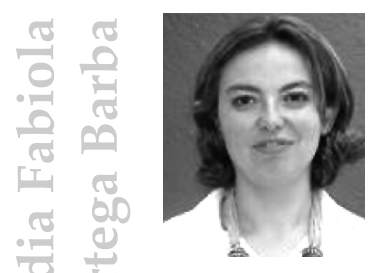

Candidata a Doctora en Pedagogía, Universidad Nacional Autónoma de México. Maestra en Ciencias de la Comunicación, Facultad de Ciencias Políticas y Sociales, Universidad Nacional Autónoma de México. Licenciada en Ciencias de la Comunicación, Facultad de Ciencias Políticas y Sociales, Universidad Nacional Autónoma de México. Académica de la Escuela de Pedagogía, especializada en temas de tecnología educativa y metodología de la investigación. Correo electrónico: [cortega@up.edu.mx].

\section{INTRODUCCIÓN}

La producción de podcast se ubica en el concepto de web $2.0^{1}$ : el usuario de la Red se convierte en productor de materiales, los cuales pone a disposición de los internautas.

Ahora bien, un material didáctico tiene como objetivo general, apoyar el proceso de enseñanza aprendizaje y como objetivos particulares mostrar conocimientos, desarrollar habilidades y mejorar comportamientos; en este sentido, un podcast deberá cumplir con éstos.

1 Si se quiere profundizar en el tema de la web 2.0 se sugiere la lectura de Cristóbal Cobo Romaní y Hugo Pardo Kuklinski, Planeta Web 2.0. Inteligencia colectiva o medios fast food? Grup de Recerca d'Interaccions Digitals, Universitat de Vic. Flacso México. Barcelona/ México. E-book de acceso gratuito en: [http://issuu.com/merce9/docs/ planeta_web2]. 
Pero, ¿qué es un podcast? Es un material, principalmente sonoro ${ }^{2} \mathrm{y}$ digital, grabado en archivos $\mathrm{mp} 3{ }^{3}, \mathrm{ogg}^{4} \mathrm{o} \mathrm{wav}^{5}$, distribuido a través de Internet por tecnología $\mathrm{RSS}^{6} \mathrm{y}$ que puede ser reproducido en una computadora o en un dispositivo móvil.

Algunas ventajas de emplear este material son:

- Difundir contenidos de forma simple sobre la plataforma web en audio.

- Dirigirse a un público específico o bien delimitado. Gracias a la naturaleza de Internet es posible alcanzar de forma cercana a públicos reducidos o con intereses específicos.

- Entregar contenido de forma regular y periódica. La lógica de emisión/suscripción en la que se basa, facilita la generación y entrega de contenidos.

Si se quiere hacer un podcast como material didáctico es necesario tomar en cuenta dos aspectos: por un lado, el didáctico y, por el otro, el de la producción audiovisual.

Específicamente atendiendo al aspecto didáctico, los productores de podcast deberán tomar en cuenta lo siguiente:

2 El podcast también hace referencia a materia audiovisual.

3 Es un sistema de compresión de audio con el cual se puede almacenar música con calidad disco compacto (CD) en 1/12 del espacio original. Las siglas MP3 responden a una abreviación de MPEG (Moving Picture Expert Group), nombre del consorcio que lo desarrolló junto con el Instituto Tecnológico Fraunhofer.

4 Ogg es un formato de archivo contenedor multimedia, desarrollado por la Fundación Xiph.org. Lo que caracteriza este formato de los anteriores (mp3 y wav) es ser abierto, diseñado para dar un alto grado de eficiencia en la visualización de archivos multimedia y la compresión de los mismos. La desventaja es la incompatibilidad con muchos reproductores.

5 Apócope de WAVE form audio format. Es un formato para almacenar sonido en archivos desarrollado en común por Microsoft e IBM. El soporte para archivos WAV fue construido en Windows 95, ello lo convirtió en un estándar de archivos de sonido en computadoras personales. Los archivos de sonido WAV terminan con la extensión «.wav» y se pueden reproducir con casi todas las aplicaciones Windows que soportan sonido. La diferencia con los archivos mp3 es que los primeros tiene un peso menor.

6 El RSS es un formato de datos utilizado para distribuir contenidos a suscriptores de un sitio web. Sus siglas en inglés son Really Simple Syndication. 
- Educando-educador: El educador es el productor del material y el educando es el receptor de los contenidos del mismo.

- Objetivos: Le proporcionan sentido al material, se trata de la finalidad del mismo, para qué será producido.

- Contenido: Éste elige el producto de acuerdo a los objetivos del material, para desarrollarlo se hace uso del guión literario.

- Metodología: En este espació se debe contemplar cómo deberá emplearse el material sonoro.

- Recursos: Para poder reproducir un podcast es necesario tener en cuenta los recursos que se necesitarán.

- Tiempo y lugar: El productor del material sonoro debe tener idea de cuándo y dónde sugerir su uso.

Al pasar al proceso formal de producción debemos atender a cuatro etapas: pre producción, producción, post producción y publicación. A continuación se describe cada una.

\section{PRE PRODUCCIÓN}

Esta etapa incluye al diagnóstico y a la planeación, pues para producir cualquier material didáctico es necesario identificar una situación educativa para la cual servirá (en este caso el podcast). Por lo anterior, el desarrollador de podcast deberá enumerar los objetivos del material y generar una temática del mismo.

El siguiente paso es la redacción del guión literario, pero antes de empezar a escribirlo, el realizador debe ubicar el tipo de podcast que producirá. A continuación se enumeran algunos tipos de podcast educativos: 
- Audio libro. Transforma la experiencia de la lectura; funciona para apoyar a débiles visuales, e inclusive, diversifica los materiales de acuerdo a los distintos estilos de aprendizaje.

- Programa de entrevistas. Permiten escuchar a uno o varios especialistas sobre un tema.

- Ponencias grabadas. Almacenan información interesante sobre algún tema.

- Cátedra magistral. Es útil como material de archivo y de ayuda a la memoria. Consiste en una grabación en vivo que después se guarda y se coloca a disposición del público a través de la Red.

- Manuales o instructivos. Este formato sirve para procesos tutoriales en donde se requiere presentar pasos a seguir.

- Clase. Éste es un formato de gran utilidad para la nivelación de contenidos entre los alumnos de un aula, pues permite guardar las sesiones ya impartidas y ponerlas a disposición de quienes no estuvieron presentes.

- Experiencias estudiantiles. Desarrollado por alumnos, generan una experiencia enriquecedora en cuanto al uso de algunas tecnologías informáticas.

El guión literario tiene como función recabar y ordenar de manera lógica el contenido del podcast en un documento similar a una monografía, para más tarde convertirlo en guión técnico. El guión literario es el compendio de información recopilada para el podcast, ésta puede ser información documental tomada de libros, revistas y documentos publicados en Internet, por citar algunos, además puede completarse el contenido a través del trabajo de campo (específicamente el uso de la entrevista a especialistas del tema).

Por otro lado, el guión técnico es la adaptación al lenguaje sonoro del guión literario, éste se ve reflejado en un documento en donde se especifican los diálogos del podcast además de las indicaciones de sonido.

El guión técnico permite plantear, específicamente, una estructura clara para el programa a grabar, realiza el proceso de grabación y edición 
de forma mucho más sencilla y productiva, además de estandarizar los procedimientos y nombrar o limitar las acciones a llevar a cabo.

Podemos encontrar dos tipos de formato para guión literario de audio: a una columna y a dos columnas. A continuación se presenta un ejemplo de cada; ambos fueron realizados por alumnos de Escuela de Pedagogía de la Universidad Panamericana.

\begin{tabular}{|c|c|}
\hline & $\begin{array}{c}\text { Nombre del proqrama: Sique tu ritmo. } \\
\text { Integrantes: Carolina Díaz Amador } \\
\text { Regina F. González Salinas } \\
\text { Rodrigo Nicolás Vite Lara }\end{array}$ \\
\hline $\begin{array}{l}\text { INTVERSIDAD } \\
\text { PANAMERICANA }\end{array}$ & $\begin{array}{l}\text { EVALUACIÓN DE } \\
\text { EDUCATIVO } \\
\text { Duración: } 3 \text { minutos } 56 \text { segundos } \\
\text { (aproximadamente) } \\
\text { Fecha de producción: } 20 \text { de febrero de } \\
2009 \text {. }\end{array}$ \\
\hline
\end{tabular}

\section{GUIÓN TÉCNICO PARA PODCAST}

\begin{tabular}{|c|c|c|}
\hline CONTROL & Fade in : Música, se mantiene 10 segundos y fade out & $10^{\prime \prime}$ \\
\hline LOC1 & $\begin{array}{l}\text { Hola radioescuchas, gracias por estar con nosotros en Sigue } \\
\text { tu ritmo, les habla Carolina Díaz Amador. Durante esta sesión } \\
\text { vamos a hablar de la evaluación de software educativo, para } \\
\text { esto entrevistaremos a la maestra en Ciencias de la } \\
\text { comunicación Claudia Fabiola Ortega Barba, quien es } \\
\text { profesora de la Facultad de Pedagogía de la Universidad } \\
\text { Panamericana. }\end{array}$ & $10^{\prime \prime}$ \\
\hline LOC1 & Hola Claudia, ¿cómo se define un software educativo? & $5^{\prime \prime}$ \\
\hline LOC2 & Respuesta & $20^{\prime \prime}$ \\
\hline LOC1 & $\begin{array}{l}\text { ¿Puede un software didáctico sustituir en su totalidad a un } \\
\text { curso impartido directamente por un docente? ¿Por qué? }\end{array}$ & $5^{\prime \prime}$ \\
\hline LOC2 & Respuesta & $20^{\prime \prime}$ \\
\hline LOC1 & $\begin{array}{l}\text { ¿Qué características debe tener un software para que sea } \\
\text { considerado útil? }\end{array}$ & $5^{\prime \prime}$ \\
\hline LOC2 & Respuesta & $20^{\prime \prime}$ \\
\hline LOC1 & $\begin{array}{l}\text { ¿Cómo influye la aplicación del software en el aprendizaje del } \\
\text { educando? }\end{array}$ & $5^{\prime \prime}$ \\
\hline LOC2 & Respuesta & $20^{\prime \prime}$ \\
\hline LOC1 & $\begin{array}{l}\text { ¿Qué ventajas da al educando aprender por medio de } \\
\text { software? }\end{array}$ & $5^{\prime \prime}$ \\
\hline LOC2 & Respuesta & $20^{\prime \prime}$ \\
\hline LOC1 & $\begin{array}{l}\text { ¿Importa más la creatividad en la elaboración del software o } \\
\text { la cantidad de información incluida? ¿Por qué? }\end{array}$ & $5^{\prime \prime}$ \\
\hline LOC2 & Respuesta & $20^{\prime \prime}$ \\
\hline LOC1 & $\begin{array}{l}\text { ¿Cuál debe ser la relación entre tiempo de } \\
\text { de sesi ón con el docente? }\end{array}$ & $5^{\prime \prime}$ \\
\hline LOC2 & Respuesta & $20^{\prime \prime}$ \\
\hline
\end{tabular}

CONTINÚA 
Ejemplo de guión a dos columnas.

\begin{tabular}{|c|c|}
\hline UNIVERSIDAD $_{\text {PANAMERICANA }}$ & $\begin{array}{r}\text { Hoja 1 } \\
\text { SIGUE TU RITMO } \\
\text { Andrea Diego Armida } \\
\text { Ana Elguero Capetillo } \\
\text { Raquel Sánchez Ávalos } \\
\text { Estrategias de aprendizaje } \\
\text { Duración: } 3 \text { minutos } \\
\text { Fecha: febrero, 2008 }\end{array}$ \\
\hline INDICACIONES TÉCNICAS & AUDIO \\
\hline \multirow[t]{2}{*}{$\begin{array}{l}\text { Entra música } 5 \text { segundos. } \\
\text { I want to break free (Queen). } \\
\text { Enseguida bajar volumen. } \\
\text { Entran locutoras. }\end{array}$} & \\
\hline & $\begin{array}{l}\text { LOCUTOR 1: ¡Hola, bienvenidos una vez más a nuestro programa! Para } \\
\text { los que ya nos conocen, yo soy Raquel Sánchez y mi compañera... } \\
\text { LOCUTOR 2: Ana Elguero. ¡Hola, Raquel! } \\
\text { LOCUTOR 1: En el programa del día de hoy, tenemos el honor de conversar } \\
\text { con la doctora Sara Galbán, cuya experiencia nos proporcionará una } \\
\text { visión práctica sobre el aprendizaje dentro del aula de clase. } \\
\text { LOCUTOR 2: Y, ¿cómo no lo sería si posee una trayectoria extensa? La } \\
\text { doctora es egresada de la Universidad Panamericana (lectura del currículum). } \\
\text { LOCUTOR 1: Un currículum impresionante, ¿no? } \\
\text { LOCUTOR 2: Sí, bastante. Pues entonces, vamos a conversar con ella, ¿les } \\
\text { parece? Raquel, te cedo la palabra... }\end{array}$ \\
\hline $\begin{array}{l}\text { Subir volumen música } \\
\text { (1.20 a } 1.33 \text { segundos. } \\
\text { Rock'n high school) } \\
\text { Bajarla hasta desaparecer. } \\
\text { Enseguida, entran locutoras. }\end{array}$ & \\
\hline
\end{tabular}

CONTINÚA...

Es importante tomar en cuenta los recursos sonoros como: la palabra hablada, la música, el silencio, los efectos y las indicaciones técnicas. En un guión pueden utilizarse las siguientes indicaciones:

\section{Caretas}

Espacio breve, donde generalmente se usan música o efectos, y también texto, que se incluye al principio y al final de un programa para personalizarlo.

Fade in Entrada de la música que sube de volumen.

Segundo plano

Se escucha de fondo. 


\begin{tabular}{l|l}
\hline Número de locutor & Loc. 1, 2.. \\
\hline Fade out & Música que baja de volumen hasta desaparecer. \\
\hline Entrevistado & Cuando entra la voz del entrevistado. \\
\hline Entrevistador & Cuando entra la voz del entrevistador. \\
\hline Fin de la entrevista & Cuando termina de hablar el entrevistado. \\
\hline Pausa & Un espacio en el sonido. \\
\hline Se mantiene & La música que permanece. \\
\hline Cortinilla & Música de unión entre las intervenciones. \\
\hline Puente musical & Cuando se cambia de una música a otra. \\
\hline
\end{tabular}

A continuación se presenta una propuesta de formato de guión.

\section{Lineamientos para la entrega del guión técnico}

\begin{tabular}{|c|c|}
\hline $\begin{array}{l}\text { INSTITUCIÓN (EN MAYÚSCULAS) } \\
\text { Indicaciones técnicas }\end{array}$ & $\begin{array}{r}\text { HOJA NÚMERO (EN MAYÚSCULAS) } \\
\text { Nombre del programa } \\
\text { Nombre de los integrantes del equipo } \\
\text { TEMA (SUBRAYADO) } \\
\begin{array}{r}\text { Duración } \\
\text { Fecha }\end{array} \\
\text { Audio }\end{array}$ \\
\hline Indicaciones & $\begin{array}{l}\text { El discurso del audio. } \\
\text { En Mayúsculas (Entrevistado, entrevistador, } \\
\text { locutor....) } \\
\qquad \text { CONTINUA...(EN MAYÚSCULAS) } \\
\text { FIN (En la última página) }\end{array}$ \\
\hline
\end{tabular}


Algunas recomendaciones cuando se escribe un guión técnico son:

- No cortar ni abreviar palabras.

- Se escribe por un solo lado de la hoja.

- No usar símbolos.

- Debe considerarse que aproximadamente una cuartilla de guión es un minuto de programa.

- Cuando se ha concluido el guión técnico se pasa a la siguiente etapa: la producción.

\section{PRODUCCIÓN}

Para producir un podcast es necesario contemplar los recursos materiales con que nos podemos apoyar. En cuanto a recursos materiales, necesitamos tanto hardware ${ }^{7}$ como software ${ }^{8}$. Los requerimientos mínimos de hardware son: una computadora con bocinas o audífonos, micrófono y tarjeta de sonido. En relación al software precisamos una grabadora de sonido. Windows cuenta con una dentro de sus herramientas de entretenimiento.

Si el trabajo se realiza en computadora cuyo sistema operativo es Mac, puede usarse para grabar el sonido del programa de garageband. Así, una vez terminado el guión técnico se procede a la grabación del los diálogos. Otra sugerencia es grabar en un espacio silencioso y con un micrófono de buena calidad para que el audio sea claro.

El siguiente paso es elegir la música que servirá de fondo. Se recomienda, al final del podcast, abrir un espacio para los créditos donde presentar los derechos de autor o bien buscar piezas musicales en la Red que

Son las partes físicas y accesorios de la computadora.

8 Son los programas y procedimientos utilizados por la computadora para funcionar. 
cuenten con licencias Creative Commons 9. En relación a este tema, actualmente muchos productores de podcast trabajan con éstas ${ }^{10}$. Otra recomendación en relación a la música es elegir piezas sin letra, pues de lo contrario puede perderse la atención del oyente.

Cuando ya se tienen los anteriores elementos, el siguiente paso es la post producción o edición.

\section{POST PRODUCCIÓN}

Para llevar a cabo la edición del podcast, necesitamos tener listo el guión técnico pues de éste dependerá el trabajo de montaje.

Para lograr podcast de calidad y tener la oportunidad de añadir y modificar elementos es indispensable un programa editor de audio. Si puede pagarse la licencia de alguno, recomendamos el Goldwave el cual puede adquirirse en la siguiente dirección: [http:/ / www.goldwave.com/], si se quiere trabajar con un software libre se puede hacer con Audacity ${ }^{11}$, el cual puede descargarse en: [http:// audacity.sourceforge.net/].

Existen tutoriales en Internet que explican el uso de los editores. A continuación presentamos una dirección electrónica en donde encontrar un tutorial de Audacity: [http://www.poderato.com/_tutorial]. Una vez instalado el software elegido se empieza el trabajo de edición.

9 Creative Commons es el nombre proporcionado a las licencias desarrolladas por la organización del mismo nombre. Ésta fue creada para ayudar a reducir las barreras legales en relación al empleo de materiales tomados de la Red. Si quiere saber más sobre este tema se recomienda revisar el video ubicado en la siguiente dirección: [http: / / www.youtube.com/watch?v=Lg6znYkNuUQ].

${ }^{10}$ Esta dirección cuenta con espacios en Internet en donde es posible bajar música bajo las licencias Creative Commons: [http:/ / podcastellano.es/podsafe-music].

${ }^{11}$ Audacity es un programa libre y de código abierto para grabar y editar sonido. Está disponible para Mac OS X, Microsoft Windows, GNU/Linux y otros sistemas operativos. Actualmente se está trabajando con el concepto de web 3.0 basado en la portabilidad de las aplicaciones; Audacity puede guardarse como una aplicación portable en un USB y utilizarse sin instalarlo en la computadora. 


\section{PUBLICACIÓN}

Para publicar un podcast es necesario suscribirse a un sitio en el cual poder subir el archivo de audio. Por lo regular se solicitan los siguientes datos: el nombre de usuario que se desea emplear en el canal (username), una dirección de correo electrónico (e-mail) en donde enviarán la contraseña para poder ingresar al sitio. El sistema informará que el registro se completa al revisar el buzón del correo.

Existen diversos sitios para publicar podcast como:

Bliptv cuyo URL ${ }^{12}$ es [http:/ / www.blip.tv/].

Podomatic_ubicado en: [http: / / www.podomatic.com/].

A manera de cierre señalaremos que el podcast es un medio de comunicación social con potencialidades didácticas que los educadores deben explotar.

Estamos a tiempo de empezar a trabajar con este tipo de recursos, los cuales se han explotado en el mundo anglosajón, principalmente en los Estados Unidos, y ahora en el mundo hispanoparlante, específicamente en España.

${ }_{12}$ Sus siglas en inglés son Uniform Resource Locator y es la dirección electrónica de la página. 


\section{REFERENCIAS BIBLIOGRÁFICAS}

BLANCO, Sonia, El podcast: situación actual en el mundo hispano hablante, Málaga, Universidad de Málaga.

CHION, Michel, Cómo se escribe un guión, Madrid, Cátedra, 2000.

COBO, Cristóbal y PARDO, Hugo, Planeta Web 2.0. Inteligencia colectiva o medios fast food, México, UVIC-FLACSO, México, 2007.

GELADO, J.A., «Historia del podcasting» en Podcastellano, en: [http: / / www.podcastellano.com/historia-del-podcasting], 2005.

HILLIARD, Robert, Guionismo para radio, televisión y nuevos medios, México, International Thomson Editores, 2000.

Podcastellano: el portal de los podcast en: [http: / www.podcastellano.es/podcasting], fecha de con 